\title{
FOURIER COEFFICIENTS AND GROWTH OF HARMONIC FUNCTIONS
}

\author{
A. FRYANT \\ Department of Mathematics \\ Utica College of Syracuse University \\ Utica, New York 13502 \\ and \\ H. SHANKAR \\ Department of Mathematics \\ Ohio University \\ Athens, Ohio 45701
}

(Recelved October 7, 1986)

ABSTRACT. We consider Harmonic Functions, $H$, of several variables. We obtain necessary and sufficient conditions on its Fourier coefficients so that $H$ is an entire harmonic (that is, has no finite singularities) function; the radius of harmonicity in terms of its Fourier coefficients in case $H$ is not entire. Further, we obtain, in terms of its Fourier coefficients, the Order and Type growth measures, both in case $\mathrm{H}$ is entire or non-entire.

KEY WORDS AND PHRASES. Harmonic function, Spherical harmonics, Entire harmonic function, Radius of harmonicity, Fourier coefficients, Order and Type growth measures, Liouville's theorem.

1980 AMS SUBJECT CLASSIFICATION CODES. 31B05, 42A16.

1. INTRODUCTION.

Let $H_{k}$ denote the set of all homogeneous, harmonic polynomials in the $n$ variables $x_{1}, x_{2}, \ldots, x_{n}$, having real coefficients. $H_{k}$ is a vector space of dimension

$$
d_{k}=(n+2 k-2) \frac{(n+k-3) !}{k !(n-2) !}
$$

(see $[1$, p. 237], or $[2$, p. 145]). With respect to the inner product

$$
\langle f, g\rangle=\frac{1}{\omega_{n-1}} \int_{|x|=1} f(x) g(x) d \sigma_{1},
$$

where $\omega_{n-1}$ denotes the area of the unit sphere $|x|=\left(x_{1}^{2}+x_{2}^{2}+\ldots+x_{n}^{2}\right)^{\frac{1}{2}}=1$, and $d \sigma_{1}$ is the element of surface area on this sphere, let $\left\{\mathrm{Y}_{k}^{\mathrm{m}}\right\}_{\mathrm{m}=1}^{d_{k}}$ be an ortho- 
normal basis for $H_{k}$. Such orthonormal, homogeneous, harmonic polynomials $Y_{k}^{m}$ are called spherical harmonics of degree $k$. It is well known that spherical harmonics of different degrees are orthogonal. That is,

$$
\int_{|x|=1} Y_{k}^{\mu}(x) Y_{j}^{\nu}(x) d \sigma_{1}=0 \text {, if } j \neq k
$$

(see $[2, p .141])$.

Let $H$ be harmonic in a neighborhood $\Omega$ of the origin in $\mathbf{R}^{\mathrm{n}}$. That is, $H$ satisfies Laplace's equation

$$
\frac{\partial^{2} \mathrm{H}}{\partial \mathbf{x}_{1}^{2}}+\frac{\partial^{2} \mathrm{H}}{\partial \mathbf{x}_{2}^{2}}+\cdots+\frac{\partial^{2} \mathrm{H}}{\partial \mathbf{x}_{n}^{2}}=0
$$

throughout $\Omega$. If $\rho>0$ is chosen so that the sphere $|x|=\rho$ is contained in $\Omega$, then on this sphere $\mathrm{H}$ has the Fourier (spherical harmonic) expansion

$$
H(x)=\sum_{k=0}^{\infty} \sum_{m=1}^{d_{k}} a_{k m} r^{k} y_{k}^{m}(x / r), \quad r=|x|,
$$

where

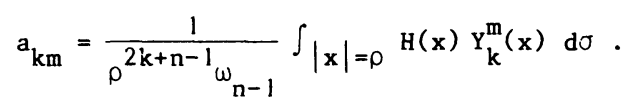

Here $d \sigma=\rho^{n-1} d \sigma$, is the element of surface area on the sphere $|x|=\rho$. The series (1.1) converges in the $L^{2}$ sense on the sphere $|x|=\rho$, and thus converges absolutely and uniformly on compact subsets of the interior ball $|x|<\rho$. The series (1.1) might, of course, converge absolutely and uniformly within larger balls centered at the origin, depending on the location of the singularities of $H$.

The objective of this paper is to characterize growth properties of $H$ explicit$1 y$ in terms of its Fourier coefficients $a_{k m}$. Specifically, necessary and sufficient conditions on the coefficients $a_{k m}$ are found so that $H$ is entire (that is, has no finite singularities), the radius of harmonicity of the spherical harmonic expansion is computed in the case where $\mathrm{H}$ is not entire, and the order and type of $\mathrm{H}$ are found in both the entire and non-entire case.

2. CONVERGENCE OF SPHERICAL HARMONIC EXPANSIONS.

The following result [2, p. 144] is of fundamental importance in our work:

THEOREM. If $\left\{\mathrm{Y}_{\mathrm{k}}^{\mathrm{m}}\right\}_{\mathrm{m}=1}^{\mathrm{d}_{\mathrm{k}}}$ is an orthonormal basis for $H_{k}$, then

$$
\sum_{m=1}^{d_{k}}\left[Y_{k}^{m}(x)\right]^{2}=\frac{d_{k}}{w_{n-1}}
$$

for all $x$ on the unit sphere $|x|=1$.

Since $d_{k}=(n+2 k-2) \frac{(n+k-3) !}{k !(n-2) !}$, and $\omega_{n-1}=\frac{2 \pi^{n / 2}}{\Gamma(n / 2)}$, we have

$$
\sum_{m=1}^{d_{k}}\left[Y_{k}^{m}(x)\right]^{2}=\frac{\Gamma(n / 2)(n+2 k-2)(n+k-3) !}{2 \pi^{n / 2} k !(n-2) !},|x|=1 \text {. }
$$

This result is a natural generalization of the Pythagorean identity 


$$
\sin ^{2} \theta+\cos ^{2} \theta=1 \text {. }
$$

For, in $\mathbf{R}_{-\frac{1}{2}}^{2}$ we have $d_{-\frac{1}{2}}=2$, and $\omega_{1}=2 \pi$. The two spherical harmonics of degree $k$ are $\pi^{-\frac{1}{2}} \sin k \Theta, \pi^{-\frac{1}{2}} \cos k \Theta$, and the result of the preceding theorem becomes

$$
\left(-\frac{1}{\sqrt{\pi}} \sin k \theta\right)^{2}+\left(\frac{1}{\sqrt{\pi}} \cos k \Theta\right)^{2}=\frac{2}{2 \pi} .
$$

Let $a_{k}=\left(a_{k 1}, a_{k 2}, \ldots, a_{k d_{k}}\right)$ be the vector of Fourier coefficients of the terms of degree $k$ in the expansion (1.1). We define

$$
\left|a_{k}\right|=\left(a_{k 1}^{2}+a_{k 2}^{2}+\ldots+a_{k d_{k}}^{2}\right)^{\frac{1}{2}} \text {. }
$$

Our first result gives the radius of harmonicity of the series (1.1) in terms of these $\left|a_{k}\right|$.

THEOREM 1. The series

$$
\sum_{k=0}^{\infty} \sum_{m=1}^{d_{k}} a_{k m} r^{k} Y_{k}^{m}(x / r),|x|=r,
$$

converges absolutely and uniformly on compact subsets of the disk $|\mathbf{x}|<R$, where $R$ is given by

$$
\mathrm{R}^{-1}=1 \lim _{\mathrm{k} \rightarrow \infty} \sup _{\mathrm{k}}\left|\mathrm{a}_{\mathrm{k}}\right|^{1 / \mathrm{k}}
$$

Further, such convergence obtains within no larger ball centered at the origin.

PROOF.

$$
\begin{aligned}
\left|\sum_{k=0}^{\infty} \sum_{m=1}^{d_{k}} a_{k m} r^{k} Y_{k}^{m}(x / r)\right| & \leq \sum_{k=0}^{\infty} r^{k}\left|\sum_{m=1}^{d_{k}} a_{k m} Y_{k}^{m}(x / r)\right| \\
& \leq \sum_{k=0}^{\infty}\left(\sum_{m=1}^{d_{k}} a_{k m}^{2}\right)^{\frac{1}{2}}\left(\sum_{m=1}^{d_{k}}\left[Y_{k}^{m}(x)\right]^{2}\right)^{\frac{1}{2}} \\
& =\sum_{k=0}^{\infty}\left|a_{k}\right|\left(d_{k} / \omega_{n-1}\right)^{\frac{1}{2}} r^{k} \\
& =\omega_{n-1}^{-\frac{1}{2}} \sum_{k=0}^{\infty} \sqrt{d_{k}}\left|a_{k}\right| r^{k} .
\end{aligned}
$$

Now,

$$
\begin{aligned}
\lim _{k \rightarrow \infty}\left(\sqrt{d_{k}}\right)^{1 / k} & =\lim _{k \rightarrow \infty}\left[(n+2 k-2) \frac{(n+k-3) !}{k !(n-2) !}\right]^{1 / 2 k} \\
& =\lim _{k \rightarrow \infty}\left[\frac{(n+k-3) !}{(k-1) !}\right]^{1 / 2 k} .
\end{aligned}
$$

Further, since $\Gamma(k+\alpha) / \Gamma(k+\beta) \sim k^{\alpha-\beta}$, the above limit is

$$
=\lim _{k \rightarrow \infty}\left(k^{n-2}\right)^{1 / 2 k}=1 \text {. }
$$

Thus the power series $\sum_{k=0}^{\infty} \sqrt{d_{k}}\left|a_{k}\right| r^{k}$ has radius of convergence $R$, where

$$
R^{-1}=1 \operatorname{imsup}_{k \rightarrow \infty}\left(\sqrt{d_{k}}\left|a_{k}\right|\right)^{1 / k}=1 \operatorname{im}_{k \rightarrow \infty} \sup _{k}\left|a_{k}\right|^{1 / k} .
$$


Since this series dominates the spherical harmonic series $\sum_{k=0}^{\infty} \sum_{m=1}^{d_{k}} a_{k m} r^{k} Y_{k}^{m}(x / r)$, the spherical harmonic series converges absolutely and uniformly for all $x$ such that $|x| \leq R-\varepsilon, \varepsilon>0$, and thus on compact subsets of the ball $|x|<R$.

To see that such convergence cannot obtain within any larger disk centered at the origin, consider

$$
M_{2}(r)=\left(\frac{1}{\omega_{n-1} r^{n-1}} \int_{|x|=r}\left[\sum_{m=0}^{\infty} \sum_{k=1}^{d_{k}} a_{k m} r^{k} Y_{k}^{m}(x / r)\right]^{2} d \sigma\right)^{\frac{1}{2}} .
$$

If the spherical harmonic series converges uniformly for $|x|=r$, then term by term integration yie1ds

$$
\begin{aligned}
& {\left[M_{2}(r)\right]^{2}=\sum_{k=0}^{\infty} \sum_{m=1}^{d_{k}} a_{k m}^{2} r^{2 k} \frac{1}{\omega_{n-1} r^{n-1}} \int|x|=r\left[y_{k}^{m}(x / r)\right]^{2} d \sigma} \\
& =\sum_{k=0}^{\infty} r^{2 k} \sum_{m=1}^{d_{k}} a_{k m}^{2} \frac{1}{\omega_{n-1}} \int_{|x|=1}\left[Y_{k}^{m}(x)\right]^{2} d \sigma_{1} \\
& =\sum_{k=0}^{\infty} r^{2 k} \sum_{m=1}^{d_{k}} a_{k m}^{2} \\
& =\sum_{k=0}^{\infty}\left|a_{k}\right|^{2} r^{2 k}
\end{aligned}
$$

This series then must be convergent, which implies

$$
\mathbf{r}^{-1} \geq \lim \sup _{k \rightarrow \infty}\left|a_{k}\right|^{1 / k}=R^{-1} \text {. }
$$

Thus $r \leq R$, and the proof of our theorem is complete.

COROLLARY. If $\mathrm{H}(\mathrm{x})=\sum_{\mathrm{k}=0}^{\infty} \sum_{\mathrm{m}=0}^{\mathrm{d}_{\mathrm{k}}} a_{\mathrm{km}} \mathrm{r}^{\mathrm{k}} \mathrm{Y}_{\mathrm{k}}^{\mathrm{m}}(\mathrm{x} / \mathrm{r})$ is harmonic in a neighborhood of the origin, then the distance $R$ from the origin to the nearest singularity of $H$ is given by $\mathrm{R}^{-1}=1 \operatorname{im}_{\mathrm{k} \rightarrow \infty} \sup _{\mathbf{k}}\left|\mathrm{a}_{\mathbf{k}}\right|^{1 / \mathrm{k}}$.

PROOF. By Harnack's theorem, the uniform limit of a sequence of harmonic functions is harmonic. Thus our previous theorem ensures that $H$ is harmonic in the open ball $|x|<R$, where $R^{-1}=1 \underset{k \rightarrow \infty}{\operatorname{imsup}}\left|a_{k}\right|^{1 / k}$. Further, $H$ cannot be harmonic throughout any larger open ball centered at the origin. For if this were the case, there would exist a $\rho>R$ such that $H$ would be harmonic on the closed ball $|x| \leq \rho$ (that is, harmonic throughout an open set.containing the closed ball $|x| \leq \rho$ ).

Since the spherical harmonics are complete in the space of square integrable functions on any sphere $|x|=\rho$ (see [2, chap. 4, sec. 2]), H would then have the Fourier series expansion

$$
H(x)=\sum_{k=0}^{\infty} \sum_{m=0}^{d_{k}} a_{k m} r^{k} Y_{k}^{m}(x / r),
$$

where the series was at least $L^{2}$ convergent on the sphere $|x|=\rho$, and uniformly convergent on compact subsets of the interior ball $|\mathbf{x}|<\rho$. But this contradicts the result of our previous theorem. Thus $H$ must have at least one singularity somewhere on the sphere $|\mathbf{x}|=\mathrm{R}$. 
In view of the result of this corollary, it is natural to call $R$ the radius of harmonicity of $\mathrm{H}$.

As an aid in investigating the growth of harmonic functions $H$, we next obtain analytic functions of $r$ which provide good upper and lower bounds on $M(r) \equiv$ $M(r, H)=\max _{|\mathbf{x}|=\mathbf{r}}|\mathrm{H}(\mathbf{x})|$.

THEOREM 2. If $H(x)=\sum_{k=0}^{\infty} \sum_{m=1}^{d_{k}} a_{k m} r^{k} y_{k}^{m}(x / r)$, then

$$
M_{2}(r) \leq M(r) \leq M(r)
$$

for all $r$ less than the radius of harmonicity $R$ of $H$, where

$$
M_{2}(r)=\left(\sum_{k=0}^{\infty}\left|a_{k}\right|^{2} r^{2 k}\right)^{\frac{1}{2}},
$$

and

$$
M(r)=\omega_{n-1}^{-1} \sum_{k=0}^{\infty} \sqrt{d_{k}}\left|a_{k}\right| r^{k} .
$$

PROOF. Derivation of the upper bound $M(r) \leq M(r)$ is contained in the proof of Theorem 1. For the lower bound, we have

$$
\begin{aligned}
M_{2}(r) & =\left(\sum_{k=0}^{\infty}\left|a_{k}\right|^{2} r^{2 k}\right)^{\frac{1}{2}} \\
& =\left[\frac{1}{\omega_{n-1} r^{n-1}} \int|x|=r H^{2}(x) d \sigma\right]^{\frac{1}{2}} \\
& \left.\leq \frac{1}{\omega_{n-1} r^{n-1}} \int_{|x|=r} d \sigma\right)^{\frac{1}{2}} \max _{|x|=r} H(x) \\
& =M(r) .
\end{aligned}
$$

3. ENTIRE HARMONIC FUNCTIONS

The results of the previous section allow for the characterization of entire harmonic functions in terms of their spherical harmonic coefficients. That is, a harmonic function having the series expansion (1.1) is entire if and only if $R=\infty$, where $R^{-1}=1 \underset{k \rightarrow \infty}{\operatorname{map}}\left|a_{k}\right|^{1 / k}$. In this section we characterize the growth of entire harmonic functions explicitly in terms of the functions' spherical harmonic coefficients. As measures of growth we use the classical concepts of order and type.

The order $\rho$ of an entire harmonic function $H$ is defined by

$$
\rho=1 \lim _{r \rightarrow \infty} \sup \frac{\log \log M(r)}{\log r},
$$

where $M(r)=M(r, H)$ is as given in the previous section. If $H$ is of $f$ inite, nonzero order, then the type $\tau$ of $H$ is defined by

$$
\tau=1 \operatorname{imsup}_{r \rightarrow \infty} \frac{\log M(r)}{r^{\rho}} .
$$

THEOREM 3. If $H(x)=\sum_{k=0}^{\infty} \sum_{m=1}^{d_{k}} a_{k m} r^{k} Y_{k}^{m}(x / r)$, is entire, then the order of $H$ is given by 


$$
\rho=1 \operatorname{imsup}_{k \rightarrow \infty} \frac{k \log k}{\log \left|a_{k}\right|^{-1}} \text {. }
$$

PROOF. Let $M_{2}(r)$ and $M(r)$ be as defined in Theorem 2. Since $H$ is entire, $\underset{k \rightarrow \infty}{\operatorname{im} \sup }\left|a_{k}\right|^{1 / k}=0$. Thus the analytic continuation of $\left[M_{2}(r)\right]^{2}$, that is

$$
\left[M_{2}(z)\right]^{2}=\sum_{k=0}^{\infty}\left|a_{k}\right|^{2} z^{2 k},
$$

is an entire analytic function of the complex variable $z$. Further, $\left|M_{2}(z)\right|^{2} \leq$ $\left[M_{2}(r)\right]^{2}$, where $r=|z|$. A1so,

$$
\begin{aligned}
\lim \sup _{r \rightarrow \infty} & \frac{\log \log \left[M_{2}(r)\right]^{2}}{\log r}=\lim \sup _{r \rightarrow \infty} \frac{\log \log M_{2}(r)}{\log r} \\
& \leq \underset{r \rightarrow \infty}{\lim \sup } \frac{\log \log M(r)}{\log r}=\rho(H) .
\end{aligned}
$$

Thus the order of $\left[M_{2}(z)\right]^{2}$ is less than or equal to the order $\rho(H)$ of $H$. But the order of an entire analytic function $f(z)=\sum_{k=0}^{\infty} c_{k} z^{k}$ is given by

$$
\rho(f)=1 \operatorname{imsup}_{k \rightarrow \infty} \frac{k \log k}{\log \left|c_{k}\right|^{-1}},[2, p .9] \text {. }
$$

We conclude that

$$
\limsup _{k \rightarrow \infty} \frac{2 k \log 2 k}{\log \left|a_{k}\right|^{-2}}=\lim \sup _{k \rightarrow \infty} \frac{k \log k}{\log \left|a_{k}\right|^{-1}}<\rho(H) .
$$

$M(r)$ is also an analytic function of $r$, and thus can be continued to complex values of its variable. We have

$$
M(z)=\omega_{n-1}^{-1} \sum_{k=0}^{\infty} \sqrt{d_{k}}\left|a_{k}\right| z^{k}
$$

Now,

$$
\lim _{k \rightarrow \infty}{\sqrt{d_{k}}}^{\frac{1}{2}}=1
$$

as was shown in the proof of Theorem 1. Thus since $H$ is entire, $M(z)$ is also an entire function. Appealing to the result of Theorem 2, we have $M(r) \leq M(r)$, and since $M(r) \leq \max _{|z|=r}|M(z)|$, it follows that the order of $H$ is less than or equal to the order of $M(z)$. But according to the classical function theoretic result [2, p. 9] the order of $M(z)$ is

$$
\lim _{k \rightarrow \infty} \sup \frac{k \log k}{\log \left(\sqrt{d_{k}}\left|a_{k}\right|\right)^{-1}}=\lim \sup _{k \rightarrow \infty} \frac{k \log k}{\log \left|a_{k}\right|^{-1}} .
$$

Therefore,

$$
\rho(H) \leq \lim \sup _{k \rightarrow \infty} \frac{k \log k}{\log \left|a_{k}\right|^{-1}},
$$


and the proof of our theorem is complete.

Note that in the preceding argument we actually show that

$$
\rho\left[M_{2}^{2}(z)\right]=\rho(H)=\rho[M(z)] .
$$

That is, the analytic functions of $r$ given in Theorem 2 which provide upper and lower bounds on $M(r)$ have the same order as $H$. They also have the same type as $H$, as is shown in the proof of our next theorem.

THEOREM 4. If $\mathrm{H}(\mathrm{x})=\sum_{\mathrm{k}=0}^{\infty} \sum_{\mathrm{m}=1}^{d_{k}} a_{k m} \mathrm{r}^{k} \mathrm{Y}_{k}^{\mathrm{m}}(\mathrm{x} / \mathrm{r})$ is an entire harmonic function of finite, non-zero order $\rho$, then the type $\tau$ of $H$ is given by

$$
\tau=(e \rho)^{-1} 1 \operatorname{imsup}_{k \rightarrow \infty} k\left|a_{k}\right|^{\rho / k} .
$$

PROOF. Since $\left[M_{2}(z)\right]^{2}$ has order $\rho$, by definition its type is

$$
\begin{aligned}
& \lim \sup _{r \rightarrow \infty} \frac{\log \left[\max _{|z|=r}\left|M_{2}(z)\right|^{2}\right]}{r^{\rho}} \\
& =2 \lim \sup _{r \rightarrow \infty} \frac{\log \left[\max _{|z|=r}\left|M_{2}(z)\right|\right]}{r^{\rho}} \\
& \leq 2 \lim \sup _{r \rightarrow \infty} \frac{\log M(r)}{r^{\rho}} \text {, }
\end{aligned}
$$

where as before $M(r)=\max |H(x)|$. Further, by the classical function theoretic result [3, p. 11] the type of an entire analytic function $f(z)=\sum_{k=0}^{\infty} c_{k} z^{k}$ having order $\rho$ is

Thus the type of $\left[M_{2}(z)\right]^{2}$ is

$$
\tau(f)=(e \rho)^{-1} 1 \operatorname{imsup}_{k \rightarrow \infty} k\left|c_{k}\right|^{\rho / k} .
$$

$$
(e \rho)^{-1} \lim \sup _{k \rightarrow \infty} 2 k\left(\left|a_{k}\right|^{2}\right)^{\rho / k}=2(e \rho)^{-1} \underset{k \rightarrow \infty}{1 i m} \sup k\left|a_{k}\right|^{\rho / k},
$$

and the previous inequality implies

$$
(e \rho)^{-1} 1 \operatorname{imsup}_{k \rightarrow \infty} k\left|a_{k}\right|^{\rho / k} \leq \tau(\mathrm{H}) .
$$

Also, since the order of $M(z)$ is $\rho$ and $M(r) \leq M(r) \leq \max _{|z|=r}|M(z)|$, we have

$$
\begin{aligned}
\tau(H) & \leq(e \rho)^{-1} 1 \lim _{k \rightarrow \infty} \sup _{k}\left(\omega_{n-1}^{-1} \sqrt{d_{k}}\left|a_{k}\right|\right)^{\rho / k} \\
& =(e \rho)^{-1} 1 \operatorname{imsup}_{k \rightarrow \infty} k\left|a_{k}\right|^{\rho / k} .
\end{aligned}
$$

4. GROWTH OF HARMONIC FUNCTIONS IN SPHERES

We next consider the growth of non-entire harmonic functions interior to the largest sphere centered at the origin which omits the function's singularities. If

$$
H(x)=\sum_{k=0}^{\infty} \sum_{m=0}^{d_{k}} a_{k m} r^{k} Y_{k}^{m}(x / r) \text {, and }
$$




$$
\mathrm{R}^{-1}=1 \operatorname{imsup}_{\mathrm{k} \rightarrow \infty}\left|\mathrm{a}_{\mathrm{k}}\right|^{1 / \mathrm{k}}, \mathrm{R} \neq 0, \infty \text {, }
$$

then we define the order $\rho$ and type $\tau$ of $H$ interior to the open ball $|\mathbf{x}|<R$ by

$$
\rho=1 \operatorname{imsup}_{r \rightarrow R} \frac{\log ^{+} \log ^{+} M(r)}{\log [R /(R-r)]}
$$

and

$$
\tau=1 \operatorname{im}_{r \rightarrow R} \sup _{[R /(R-r)]^{\rho}} \frac{\log ^{+} M(r)}{[R /(R<\rho<\infty,}
$$

where $M(r)=\max _{|x|=r}|H(x)|$. The definitions agree with those for the order and type of non-entire analytic functions (see [4]). Further, if $f(z)=\sum_{k=1}^{\infty} c_{k} z^{k}$, where

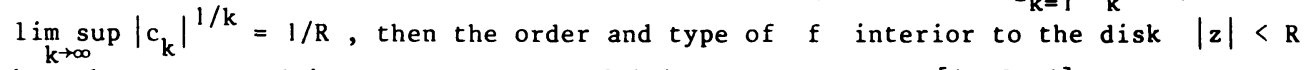
have been computed in terms of the coefficients $c_{k}$ of $f[4,5,6]$. These results are

$$
\frac{\rho}{\rho+1}=1 \limsup _{k \rightarrow \infty} \frac{\log ^{+} \log ^{+}\left|c_{k}\right| R^{k}}{\log k}
$$

and

$$
\tau=\frac{\rho^{\rho}}{(\rho+1)^{\rho+1}} \lim _{k \rightarrow \infty} \sup \frac{\left(\log ^{+}\left|c_{k}\right| R^{k}\right)^{\rho+1}}{k^{\rho}} .
$$

Arguing as in the proofs of Theorems 3 and 4 , we obtain such results for harmonic functions:

THEOREM 5. If $\mathrm{H}(\mathrm{x})=\sum_{\mathrm{k}=0}^{\infty} \sum_{\mathrm{m}=1}^{\mathrm{d}_{k}} a_{k m} \mathrm{r}^{\mathrm{k}} \mathrm{Y}_{k}^{\mathrm{m}}(\mathrm{x} / \mathrm{r})$, where $\lim _{\mathrm{k} \rightarrow \infty} \sup \left|a_{k}\right|^{1 / k}=1 / \mathrm{R}$, $R \neq 0, \infty$, then the order $\rho$ of $H$ is given by

$$
\frac{\rho}{\rho+1}=1 \operatorname{imsup}_{k \rightarrow \infty} \frac{\log ^{+} \log ^{+}\left|a_{k}\right| R^{k}}{\log k},
$$

and the type $\tau$ of $H$ is

$$
l=\frac{\rho^{\rho}}{(\rho+1)^{\rho+1}} \lim _{k \rightarrow \infty} \sup \frac{\left(\log ^{+}\left|a_{k}\right| R^{k}\right)^{\rho+1}}{k^{\rho}} .
$$

The proof of this theorem is obtained by again appealing to the analytic bounds on $M(r)$ provided by Theorem 2 .

5. LIOUVILLE'S THEOREM

A very simple proof of the analog of Liouville's theorem for entire harmonic functions can be given by arguing on the function $M_{2}(r)$ defined in Theorem 2 . We give this proof below.

THEOREM 6. If $H$ is an entire harmonic function in $\mathbf{R}^{n}$, and $|H(x)| \leq B$ for a11 $x \in \mathbf{R}^{\mathrm{n}}$, then $\mathrm{H}$ is constant.

PROOF. If $H$ is entire, then it has the spherical harmonic expansion

$$
H(x)=\sum_{k=0}^{\infty} \sum_{m=1}^{d_{k}} a_{k m} r^{k} Y_{k}^{m}(x / r),
$$

and the series converges uniformly on compact subsets of $\mathbf{R}^{\mathbf{n}}$. Thus 


$$
\begin{aligned}
{\left[M_{2}(r)\right]^{2} } & =\frac{1}{\omega_{n-1} r^{n-1}} \int|x|=r[H(x)]^{2} d \sigma \\
& =\sum_{k=0}^{\infty}\left|a_{k}\right|^{2} r^{2 k} \text {, for all } r>0 .
\end{aligned}
$$

Now, suppose $|H(x)| \leq B$ for all $x \in \mathbf{R}^{n}$. Then since $M_{2}(r) \leq \max _{|\mathbf{x}|=\mathbf{r}}|H(x)|$, we have $\sum_{k=0}^{\infty}\left|a_{k}\right|^{2} r^{2 k} \leq B^{2}$ for $a 11 \quad r>0$. Thus $\left|a_{k}\right|^{2} r^{2 k} \leq B^{2}$ for $a 11 r>0$, which implies $\left|a_{k}\right|^{2}=0$ for $k=1,2, \ldots$. But $a_{k}^{2}=\sum_{m=1}^{d_{k}} a_{k m}^{2}$. Hence $a_{k m}=0$ for all $k \geq 1$, and all corresponding values of $m$. This yeilds $H(x) \equiv a_{00}$.

For proofs of stronger analogs of Liouville's theorem for entire harmonic functions, see [7, chap. 2].

Note that the argument given here could also be used to prove the classical Liouville theorem for entire analytic functions $f(z)=\sum_{k=0}^{\infty} c_{k} z^{k}$. We believe this approach is to be preferred over that found in most presentations, owing to its wider applicability. In particular, it can be easily used to obtain analogs of Liouville's theorem for solutions of many partial differential equations. Nevertheless, to the best of our knowledge this approach to Liouville's theorem has not yet found a place in the literature.

Finally, we note that all the results of this paper hold as stated for complex valued harmonic functions in $R^{n}$; that is, for $H(x)=\sum_{k=0}^{\infty} \sum_{m=1}^{d_{k}} a_{k m} r^{k} Y_{k}^{m}(x / r)$, where the coefficients $a_{k m}$ are complex numbers. For this, only minor changes are required in the corresponding development.

6. RELATED RESULTS

Work on the growth of harmonic functions in dimensions greater than 2 has a history going back at least fifty years. In [8] and [9] the growth of harmonic functions in $\mathbf{R}^{3}$ is considered, and the Bergman $B_{3}$ integral operator is used as a principal tool in the investigation. Paper [10] contains results similar to those presented here, but for harmonic functions in $\mathbf{R}^{3}$. The growth of harmonic functions in $\mathbf{R}^{\mathrm{n}}, \mathrm{n} \geq 3$, is related to derivatives of the functions at the origin in [11, 12, 13]. Growth of entire solutions of certain elliptic partial differential equations is considered in $[14,15]$, and special cases of these equations yield axisymmetric harmonic functions in $\mathbf{R}^{\mathrm{n}}$.

In [16] the growth of entire harmonic functions in $\mathbf{R}^{3}$ is expressed in terms of the function's coefficients, and in $\mathbf{R}^{\mathrm{n}}$ in terms of the maximum value attained by the function's $k^{\text {th }}$ degree spherical harmonic on spheres centered at the origin.

ACKNOWLEDGEMEN'T. This paper is dedicated to Professor Morris Marden.

\section{REFERENCES}

1. ERDELYI, A. Higher Transcendental Functions, Vol. 2, McGraw-Hill, New York, 1953.

2. STEIN, E.M., and WEISS, G. Fourier Analysis on Euclidean Spaces, Princeton University Press, Princeton, New Jersey, 1971.

3. BOAS, R.P. Entire Functions, Academic Press, New York, 1954.

4. BAGDA, R., and SHANKAR, H. On the type and lower type of analytic functions, S. Rananujan Memorial Volume Madras India, 1974.

5. MACLANE, G.R. Asymptotic Values of Holomorphic Functions, Rice University Studies, Vo1. 49, no. 1, Rice University Press, Houston, Texas, 1963. 
6. BAJPAI, S.K., TANNE, J., and WHITTIER, J. A decomposition theorem for an analytic function, J. Math. Anal. Appl., 48 (1974), 736-742.

7. PROTTER, M., and WEINBERGER, H. Maximum Principles in Differential Equations, Springer-Verlag, New York, 1984.

8. FRYANT, A. Growth of entire harmonic functions in $\mathbf{R}^{3}$, J. Math. Anal. Appl., 66 (1978), 599-605.

9. TEMLIAKOW, A. Zu dem Wachstumproblem der harmonischen Functionen des drei dimensionalen Raumes, Recueil Mathematique, 42 (1935), 708-718.

10. FRYANT, A. Spherical harmonic expansions, Pure and Applied Mathematika Sciences, Pure App1. Math. Sci., 22 (1985), 25-31.

11. FRYANT, A., and SHANKAR, H. Growth of Harmonic functions in hyperspheres, J. Math. Ana1. Appl., 121 (1987) (to appear).

12. FUGARD, T.B. Growth of entire harmonic functions in $\mathbf{R}^{\mathrm{n}}$, J. Math. Anal. Appl., 74 (1980), 286-291.

13. FUGARD, T.B. On the largest ball of harmonic continuation, J. Math. Anal. Appl., $90(1982), 548-554$.

14. FRYANT, A. Growth and complete sequences of generalized axisymmetric potentials, J. Approx. Theory, 19 (1977), 361-370.

15. KAPOOR, G.P., and NAUTIYAL, A. On the growth of generalized axisymmetric potentials, Indian J. Pure Appl. Math., 13 (1982), 1240-1245.

16. VESELOVSHAYA, 0.v. Growth of entire functions which are harmonic in $\mathrm{k}^{\mathrm{n}}$, Soviet Math. (IZ.VUZ), 27 (1983), no. 10, 14-21. 


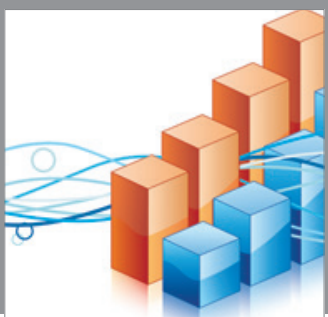

Advances in

Operations Research

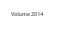

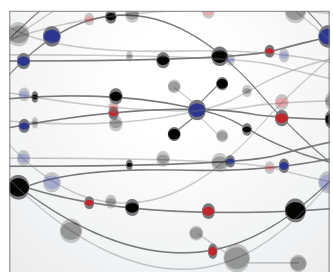

\section{The Scientific} World Journal
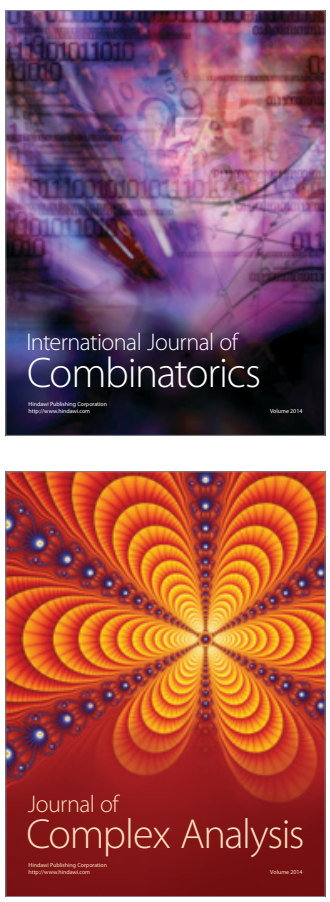

International Journal of

Mathematics and

Mathematical

Sciences
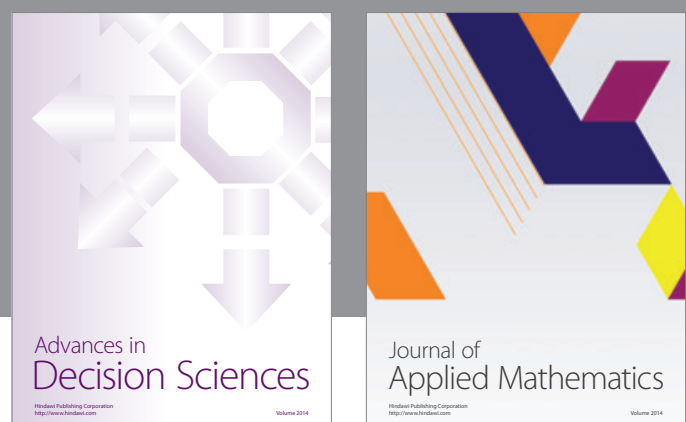

Journal of

Applied Mathematics
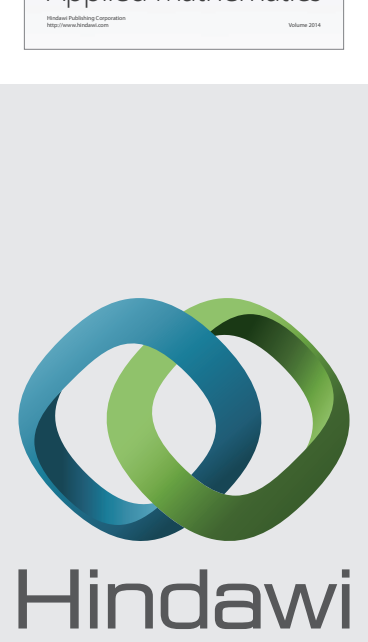

Submit your manuscripts at http://www.hindawi.com
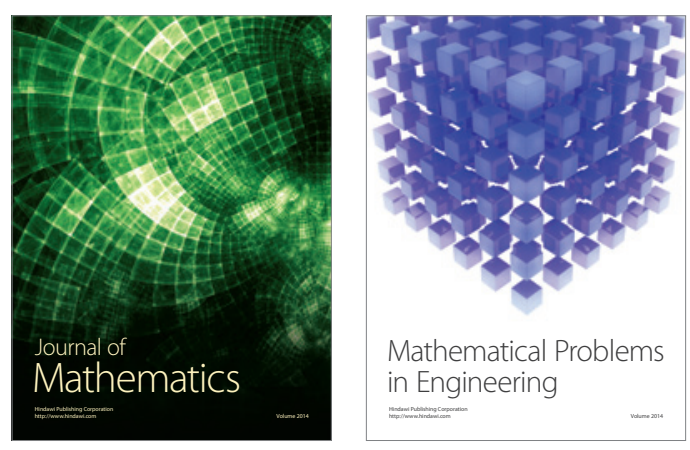

Mathematical Problems in Engineering
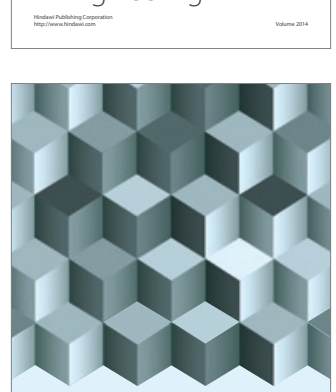

Journal of

Function Spaces
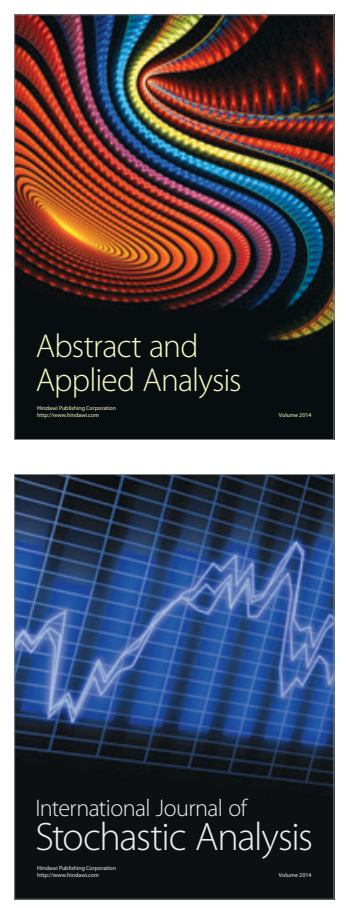

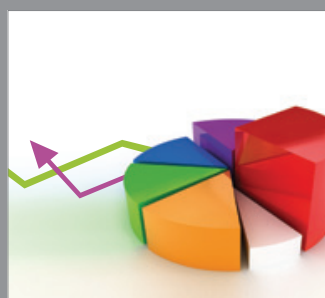

ournal of

Probability and Statistics

Promensencen
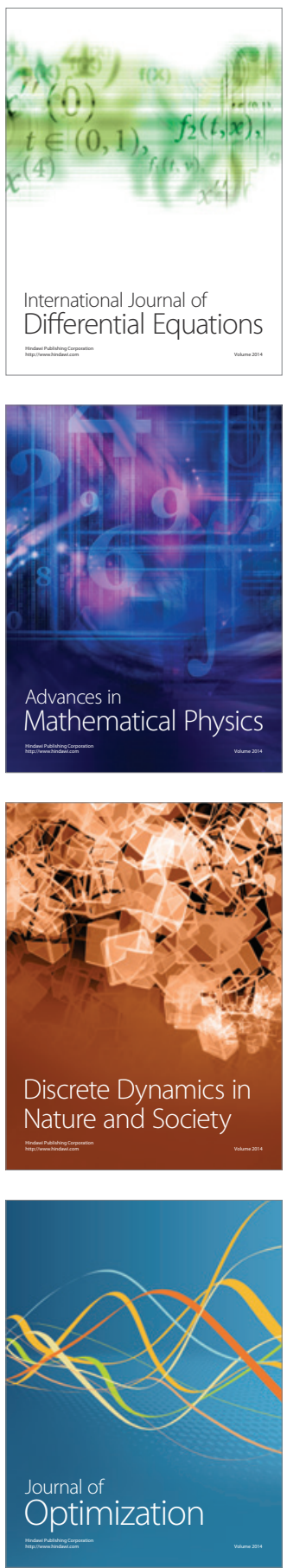